\title{
Surgical revascularization for cardiac allograft vasculopathy: Is it still an option?
}

\author{
Jay K. Bhama, MD, ${ }^{a}$ Duc Q. Nguyen, MD, ${ }^{a}$ Sun Scolieri, MD, ${ }^{\mathrm{b}}$ Jeffrey J. Teuteberg, MD, ${ }^{\mathrm{b}}$ Yoshiya Toyoda, MD, PhD, \\ Robert L. Kormos, MD, ${ }^{a}$ Kenneth R. McCurry, MD, ${ }^{a}$ Dennis McNamara, MD, ${ }^{b}$ and Christian A. Bermudez, MD ${ }^{a}$
}

\begin{abstract}
Objectives: Cardiac allograft vasculopathy remains a major cause of mortality after cardiac transplantation. Percutaneous revascularization has become the mainstay of therapy given the poor historical outcomes with surgery. Outcomes following surgical revascularization are evaluated to determine whether surgery remains a viable therapeutic option.
\end{abstract}

\begin{abstract}
Methods: A retrospective analysis was performed of 13 heart transplant recipients who had cardiac allograft vasculopathy requiring coronary artery bypass grafting with or without adjunctive percutaneous coronary intervention for revascularization from 1999 to 2008.

Results: Thirteen patients had 14 coronary artery bypass grafting procedures at $141 \pm 66$ months after transplantation. The average number of grafts was 2.3. Eight were performed without cardiopulmonary bypass, of which 5 were approached via left thoracotomy and the remainder via repeat sternotomy. One patient had renal failure and a cerebrovascular accident. Percutaneous coronary intervention before or after coronary artery bypass grafting was required in 3 patients. There were no perioperative mortalities. At mean follow-up of $39 \pm 36$ months, 3 patients have died, 2 from progressive cardiac allograft vasculopathy and 1 from lung cancer. Kaplan-Meier survival for this group of patients was $92 \%, 83 \%$, and $83 \%$ at 1,5 , and 7 years, respectively.
\end{abstract}

Conclusions: Surgical revascularization for cardiac allograft vasculopathy remains a viable treatment option for appropriate patients and may be performed safely with good medium-term outcomes. However, patients remain at risk for disease progression and may require percutaneous or surgical reintervention.

Despite recent advances in immunosuppression, cardiac allograft vasculopathy (CAV) remains a major cause of mortality after the first year of transplantation, with an incidence between $30 \%$ and $50 \%$ at 5 years. ${ }^{1}$ The mainstays of nonpharmacologic treatment options include percutaneous coronary intervention (PCI), coronary artery bypass grafting $(\mathrm{CABG})$, and retransplantation. Although outcomes following retransplantation for this group of patients have improved, the scarcity of suitable donor organs remains a significant limitation, and consequently this option is usually reserved for patients with disease progression despite revascularization or those with diffuse coronary involvement. ${ }^{2}$ PCI has become the primary treatment modality for heart transplant recipients with CAV and is associated with excellent short-term results. Its applicability is limited, however, considering the diffuse and heterogeneous coronary involvement typically seen in patients with $\mathrm{CAV}^{3}$ Historically, CABG has been associated with poor surgical

From the Division of Cardiothoracic Transplantation, Heart, Lung \& Esophageal Surgery Institute, ${ }^{\mathrm{a}}$ and The Cardiovascular Institute, ${ }^{\mathrm{b}}$ University of Pittsburgh Medical Center, Pittsburgh, Pa.

Received for publication Aug 28, 2008; revisions received Dec 26, 2008; accepted for publication Feb 3, 2009.

Address for reprints: Jay K. Bhama, MD, 200 Lothrop St, Suite C-900, Heart, Lung \& Esophageal Surgery Institute, Division of Cardiothoracic Transplantation, University of Pittsburgh Medical Center, Pittsburgh, PA 15213 (E-mail: bhamajk@ upmc.edu).

J Thorac Cardiovasc Surg 2009;137:1488-92

$0022-5223 / \$ 36.00$

Copyright (c) 2009 by The American Association for Thoracic Surgery doi:10.1016/j.jtcvs.2009.02.026 outcomes with early mortality rates in excess of $30 \% .{ }^{4} \mathrm{How}-$ ever, little recent data exist in the literature regarding the outcomes of CABG in patients with CAV. We present the largest single-center experience with surgical revascularization for $\mathrm{CAV}$ and report short-term and midterm outcomes.

\section{METHODS \\ Patients}

Approval was obtained from the University of Pittsburgh Medical Center Total Quality Control council. Since the inception of our heart transplant program in 1980 through 2008, a total of 1175 patients have had orthotopic cardiac transplantation. A retrospective review was performed of 13 patients who had CAV post-heart transplantation and were treated with surgical revascularization from 1999 to 2008 . All patients were evaluated for PCI prior to surgical intervention, and 3 patients had PCI either before or after surgical revascularization. A detailed chart review was then performed for each patient to collect demographic, procedural, and outcome data. Follow-up was complete in all patients.

\section{Immunosuppression and Infection Prophylaxis}

The immunosuppressive regimen evolved over time with initial emphasis on the use of cyclosporine with either steroids or aziothioprine. In 1993, we began routinely using tacrolimus instead of cyclosporine. Our current protocol also uses mycophenolate mofetil maintenance therapy, which was introduced routinely beginning in 1999. Induction therapy with antithymocyte globulin was used selectively in some cases. All patients received Pneumocystis carinii prophylaxis with trimethoprim/sulfamethoxazole, cytomegalovirus prophylaxis with valacyclovir or valgancyclovir, and Clostridium difficile prophylaxis with oral metronidazole.

\section{Procedures}

PCI was considered the primary modality for therapy, and all patients were evaluated for suitability by an interventional cardiologist. PCI was 
$\mathrm{CABG}=$ coronary artery bypass grafting

$\mathrm{CAV}=$ cardiac allograft vasculopathy

$\mathrm{CPB}=$ cardiopulmonary bypass

PCI = percutaneous coronary intervention

\section{Abbreviations and Acronyms}
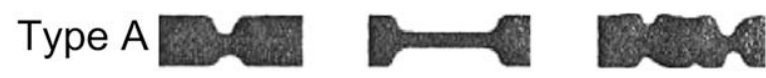

Type B

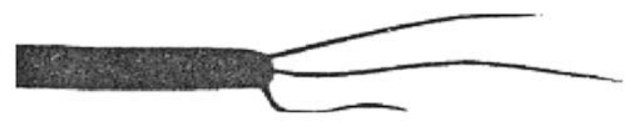

performed utilizing standard techniques, including angioplasty and/or stent deployment along with anticoagulation and antiplatelet pharmacotherapy. Procedural success was defined as residual diameter stenosis of $<50 \%$. Patients were treated with aspirin indefinitely and clopidogrel for 6 to 12 months following intervention based upon the type of procedure performed. Follow-up angiograms were not performed routinely due to the risk of contrast nephropathy. Individuals with symptoms or a noninvasive stress test suggestive of ischemia or worsening left ventricular function had angiography. Restenosis was defined as $>50 \%$ narrowing of the target lesion by visual estimation.

Surgical revascularization was favored in patients with multivessel involvement or in the presence of single-vessel disease not amenable to PCI due to technical limitations or the presence of active ischemia or anginal symptoms. Standard CABG was performed via midsternotomy utilizing right atrial-aortic cardiopulmonary bypass (CPB) with antegrade, mild hypothermia, aortic crossclamping, and antegrade/retrograde cold blood cardioplegia. Alternate approaches included revascularization via left thoracotomy or midsternotomy with off-pump CABG techniques. A left thoracotomy approach was favored for patients with single- or double-vessel disease isolated to the anterior and lateral territories.

\section{Angiographic Classification of Lesions}

Coronary angiograms were reviewed and lesions were classified according to the system of Gao and colleagues ${ }^{5}$ (Figure 1). Type A lesions were discrete and tubular or had multiple stenoses. Type B1 lesions were abrupt in onset with distal diffuse concentric narrowing and obliteration. Type B2 lesions had gradual, concentric tapering with distal portions retaining some residual lumen. Type $\mathrm{C}$ lesions had narrowed irregular distal branches with terminations often nontapered and squared off, ending abruptly. Revascularization was required in 1 patient with catheter-induced dissection of the left main coronary artery; this was classified as type A.

\section{Statistics}

Descriptive statistics are reported as mean \pm standard deviation. Survival was calculated according to the method of Kaplan and Meier with death as the primary end point.

\section{RESULTS}

Demographic data for this group of patients are listed in Table 1 . The average age at intervention was $57 \pm 15$ years (range 20-75). The mean interval between transplantation and CABG was $147 \pm 75$ months (range 15-251 months). Most patients had preserved left ventricular function and presented with angina. Triple-vessel disease was the predominant form of CAV treated with revascularization [5/13 (38\%)].

Procedural data for the 13 patients who had CABG are listed in Table 2. The average number of grafts per patients was 2.3. Off-pump techniques were utilized in 8 cases $(57 \%)$ with 5 cases $(36 \%)$ approached via a left thoracotomy. The left internal thoracic artery was used in $11 / 13$

TABLE 1. Demographic data for patients having surgical revascularization for the treatment of $\mathrm{CAV}$

\begin{tabular}{lc}
\hline \multicolumn{1}{c}{ Variable } & Value $(\mathbf{n}=\mathbf{1 3})$ \\
\hline Mean age at intervention (y) & $57 \pm 15$ \\
Male genderMale & 11 \\
Diabetes & 5 \\
Hypertension & 11 \\
Dyslipidemia & 8 \\
Mean interval to intervention (mo) & $147 \pm 75$ \\
LVEF (\%) at intervention & $53 \pm 7$ \\
Presenting symptom & \\
$\quad$ Angina & 8 \\
Asymptomatic & 4 \\
CHF & 1 \\
$\quad$ Arrhythmia & 0 \\
Preintervention myocardial infarction & 2 \\
Severity of coronary disease & \\
Single vessel & 4 \\
Double vessel & 4 \\
Triple vessel & 5 \\
\hline
\end{tabular}

$C A B G$, Coronary artery bypass graft; $C A V$, cardiac allograft vasculopathy; $C H F$, congestive heart failure; $L V E F$, left ventricular ejection fraction; $P C I$, percutaneous coronary interventions.

(85\%) patients. Type C angiographic grade lesions accounted for $31 \%$ of lesions bypassed. Three patients in this group had multiple interventions including PCI performed before, after, or as an adjunct to CABG. Specific details regarding the location of lesions and order of interventions are delineated in Table 3. One patient required repeat $\mathrm{CABG}$ for a myocardial infarction following PCI. Five of the 6 patients who had surveillance angiography had patent bypass grafts. One patient required PCI subsequent to surveillance angiography for a diseased saphenous vein graft. 
TABLE 2. Procedural data for 13 patients having CABG for the treatment of CAV

\begin{tabular}{lc}
\hline \multicolumn{1}{c}{ Variable } & Value \\
\hline Total number of procedures & $14 *$ \\
Total number of lesions bypassed & 32 \\
Angiographic grade of lesions bypassed & \\
A & 7 \\
B1 & 7 \\
B2 & 4 \\
C & 10 \\
Unknown & 4 \\
Surgical approach & \\
On-pump + repeat sternotomy & 6 \\
Off-pump + repeat sternotomy & 3 \\
Off-pump + left thoracotomy & 5 \\
Number of grafts (average) & 2.3 \\
1 & 4 \\
2 & 4 \\
3 & 4 \\
4 & 2 \\
Proportion LITA utilization & 11 \\
\hline CABG, Coronary artery bypass graft; $C A V$, cardiac allograft vasculopathy; LITA, left \\
internal thoracic artery. ${ }^{*}$ One patient had 2 operations.
\end{tabular}

Postprocedural morbidity and survival outcomes are listed in Table 4. No patient had low cardiac output or required reoperation for bleeding. There was no perioperative mortality. At a mean follow-up of $39 \pm 36$ months (range 0.3-110 months) after CABG, 10/13 (77\%) patients are alive. Two patients died of progressive CAV and 1 from lung cancer. Kaplan-Meier survival for this group of patients was $92 \%, 83 \%$, and $83 \%$ at 1,5 , and 7 years, respectively (Figure 2).

\section{DISCUSSION}

In this study, we present early to midterm outcomes with CABG for the treatment of CAV in 13 patients demonstrating that surgical management remains a viable option for selected patients. CAV remains the nemesis of cardiac transplant recipients surviving beyond the first year of transplant. In most patients, CAV is an insidious process that may occur without warning, resulting in silent myocardial infarction, arrhythmia, or sudden death. Advances in our understanding regarding CAV have focused largely upon understanding its pathogenesis and methods by which to attenuate its progres- sion. Preventative strategies are directed toward limiting both cellular and humoral rejection during the first year posttransplantation by optimization of immunosuppressive therapy. Other preventative strategies include treatment of nonimmunologic factors such as hyperlipidemia, hypertension, and cytomegalovirus infection. Novel modalities for pharmacologic therapy include immunosuppressive agents such as sirolimus or everolimus, both of which have demonstrated improvements in disease progression but have not significantly impacted overall survival. ${ }^{6-8}$ Despite these measures, CAV continues to account for approximately $30 \%$ to $50 \%$ of deaths in cardiac transplant recipients. ${ }^{1}$

The primary modalities of treatment used in the management of advanced CAV include PCI, surgical revascularization, and retransplantation. Selection among these modalities depends primarily upon the extent of disease. PCI and CABG are generally reserved for those with focal proximal disease; for most patients with diffuse coronary involvement, PCI and $\mathrm{CABG}$ remain ineffective due to inadequate anatomic features and poor distal vessel quality. For these patients, retransplantation remains the primary modality for treatment. Although earlier reports noted that the 1-year mortality for patients retransplanted for CAV was $30 \%$ to $40 \%$, more recent studies have demonstrated that in otherwise healthy patients, retransplantation may be carried out safely with outcomes that are similar to primary transplantation. ${ }^{3}$ There had been a transient interest in transmyocardial laser revascularization after initial case reports, but late follow-up has failed to demonstrate sustained benefits. ${ }^{9,10}$

In this study, which we believe to be the largest singlecenter experience described in the literature, we demonstrate significant improvement in early survival following surgical management as compared with previous studies (Table 5). However, the literature regarding standard surgical revascularization for CAV is relatively limited, with only 3 previously published series. A multi-institutional study by Halle and associates ${ }^{11}$ reported the outcomes of 12 patients who had CABG. Four patients died perioperatively due to ventricular failure in 3 patients and bleeding in 1 patient. Distal arteriopathy (type $\mathrm{C}$ ) on the preoperative angiogram was noted in $80 \%$ of patients who died versus $14 \%$ of survivors and was felt to be a significant predictor of increased mortality. This study did not delineate surgical considerations

TABLE 3. Multiple interventions in patients who had combined PCI and CABG for treatment of CAV

\begin{tabular}{|c|c|c|c|c|c|}
\hline Patient & 1st intervention & 2nd intervention & 3rd intervention & 4th intervention & 5th intervention \\
\hline \multirow[t]{2}{*}{1} & $\mathrm{CX} 1, \mathrm{CX} 2$ & LAD, D & & & \\
\hline & PCI & CABG & & & \\
\hline \multirow[t]{2}{*}{2} & $\mathrm{CX}$ and $\mathrm{LAD}$ & $\mathrm{CX}$ and $\mathrm{LAD}$ & LAD & $\mathrm{CX}$ & \\
\hline & PCI & PCI & CABG & PCI* & \\
\hline \multirow[t]{2}{*}{3} & LAD & CX and RCA & LM and CX & $\mathrm{CX}$ & SVG \\
\hline & CABG & PCI* & PCI & CABG & PCI \\
\hline
\end{tabular}

$C A B G$, Coronary artery bypass grafting; $C A V$, cardiac allograft vasculopathy; $C X$, circumflex; $D$, diagonal; $L A D$, left anterior descending; $L M$, left main; $R C A$, right coronary artery; $P C I$, percutaneous coronary intervention; $S V G$, saphenous vein graft. *Planned intervention. 
TABLE 4. Postprocedural morbidity and survival data for 13 patients having surgical revascularization for the treatment of $\mathrm{CAV}$

\begin{tabular}{lc}
\hline \multicolumn{1}{c}{ Variable } & Value \\
\hline Complication & \\
ARF & 1 \\
CVA & 1 \\
Infection (sepsis, pneumonia, urine) & 0 \\
Return to OR for bleeding & 0 \\
Arrhythmia & 0 \\
Early mortality* & 0 \\
Late mortality & \\
Cardiac & 2 \\
Noncardiac & 1 \\
\hline$A R F$, Acute renal failure; $C A V$, cardiac allograft vasculopathy; $C V A$, cerebrovascular \\
accident; $O R$, operating room. *Within 30 days or during primary hospitalization.
\end{tabular}

including the use of off-pump techniques, which may be relevant to surgical outcomes. Parry and colleagues ${ }^{12}$ described 5 patients who had CABG; 2 died perioperatively, 1 from left ventricular failure and the other from acute rejection. All 5 cases were done with normothermic $\mathrm{CPB}$ with use of the left internal thoracic artery. Musci and coworkers ${ }^{13}$ reported their results with CABG in 7 patients with CAV with 3 perioperative deaths. All patients who died had diffuse, distal arterial disease (type C) and emergency surgery.

Evaluation of the distribution and type of lesions for which CABG was performed (Table 2) revealed that 10/32 lesions bypassed $(31 \%)$ were type $\mathrm{C}$, which in previous reports has not been felt to be amenable to surgical revascularization. In our experience, these patients had similar early and midterm outcomes following surgery as patients with lesser degrees of coronary disease. We believe that this may be explained in part by the observation that many of these patients had combinations of lesions at different stages, and the most advanced lesion was the one used to classify the respective target vessel. For example, a vessel with diffuse disease (type C) might be accompanied by a proximal $80 \%$ stenosis (type A). Although this would be classified as a type $\mathrm{C}$ lesion, if the artery was of adequate size for revascularization, we considered the patient an acceptable candidate for surgery. This was the case in the 3 patients who had isolated bypass of the left anterior descending artery for type $\mathrm{C}$ disease. In addition, we also noted that several patients had type $\mathrm{C}$ disease in 1 territory but lesser disease (type A or B) in another territory. This was the case for the other 7 patients with type $\mathrm{C}$ disease who had multivessel revascularization. Therefore, the presence of hemodynamically significant lesions, even if accompanied by type $\mathrm{C}$ disease, warranted revascularization if the vessel was deemed of an adequate size (1.8 to $2 \mathrm{~mm}$ ). Although it is encouraging that all of these patients did well, we are not suggesting that surgical revascularization is always the correct answer for patients with type $\mathrm{C}$ disease. Clearly, careful patient selection is very important in achieving good outcomes, and mul-

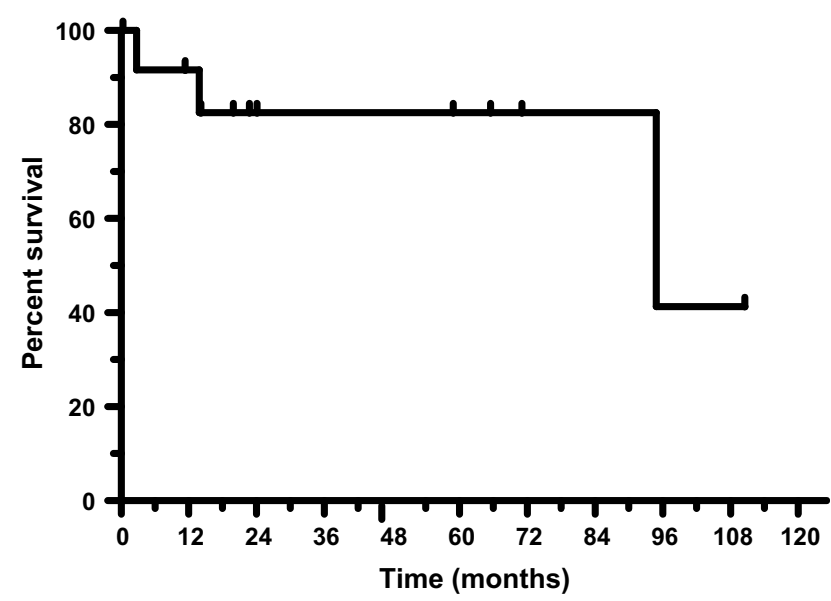

FIGURE 2. Kaplan-Meier survival for patients having surgical revascularization for the treatment of cardiac allograft vasculopathy.

tiple factors should be considered including the patient's overall physiologic and functional status, the presence of concomitant renal dysfunction, coronary anatomy (size and disease distribution), the likelihood of achieving complete revascularization, and whether alternative surgical approaches are applicable.

Our surgical strategy is unique in that we selectively utilized off-pump techniques, avoiding CPB in the majority of cases with the intent of preventing the potential deleterious effect of CPB on end-organ function. Additionally, by utilizing a left thoracotomy incision for patients requiring limited revascularization, a secondary sternotomy was avoided in $36 \%$ of cases. We believe that avoiding the inflammatory cytokine activation and deleterious effect on the coagulation cascade that typically accompanies redo cardiac surgery may be a contributing factor to the improved outcomes experienced by these relatively high-risk patients. ${ }^{14}$

There are several limitations to this study, aside from its retrospective nature. The limited number of patients and strict patient selection criteria make it difficult to determine the exact impact of the different surgical techniques on postoperative outcome. Additionally, the study spanned a 10-year period in which patients were treated with different immunosuppressive regimens. Although this may have potentially impacted the development of CAV and its

TABLE 5. Review of the literature on surgical revascularization for CAV including data regarding utilization of CPB and angiographic lesion classification

\begin{tabular}{lcccc}
\hline & \multicolumn{4}{c}{ Type C } \\
Author (year) & $\begin{array}{c}\text { Number } \\
\text { of patients }\end{array}$ & $\begin{array}{c}\text { Off-pump } \\
(\%)\end{array}$ & $\begin{array}{c}\text { lesion } \\
\text { grade (\%) }\end{array}$ & $\begin{array}{c}\text { Early } \\
\text { mortality (\%)* }\end{array}$ \\
\hline Halle et al $(1995)^{11}$ & 12 & 0 & 42 & 25 \\
Parry et al $(1996)^{12}$ & 5 & 0 & 0 & 40 \\
Musci et al (1998) & 7 & 14 & 57 & 43 \\
\hline$C A V$, Cardiac allograft vasculopathy; CPB, cardiopulmonary bypass. *Within 30 days \\
or primary hospitalization.
\end{tabular}


progression following revascularization, it is unlikely to have impacted the quality of early surgical outcomes. Although our results with surgical revascularization are encouraging, they should be interpreted with caution given the limited angiographic follow-up in only 6 of 13 patients $(46 \%)$. Although our patients experienced excellent early survival following surgical revascularization, adjunctive PCI was required in 2 patients. These findings highlight the insidious nature of CAV and demonstrate that although CABG may be a durable solution, it clearly does not represent a cure for these patients.

In conclusion, we demonstrate improved early outcomes following surgical revascularization for patients with CAV as compared with previous reports. These results may be related to a thorough preoperative assessment and the use of alternative approaches including off-pump techniques and the avoidance of secondary sternotomy, factors that may play a significant role in preventing complications including perioperative bleeding and renal deterioration. Although surgical revascularization may not be suitable for all patients with CAV, candidates with acceptable coronary anatomy should not be denied surgery based upon the risk of surgery itself. Rather, the decision to operate should be based on the usual algorithms used in selecting candidates for reoperative CABG. Close and frequent surveillance is necessary, even after successful CABG, due to the progressive nature of the disease and frequent need for reintervention.

We acknowledge the editorial assistance of Shannon L. Wyszomierski, $\mathrm{PhD}$, in the preparation of this manuscript.

\section{References}

1. Taylor DO, Edwards LB, Boucek MM, Trulock EP, Keck BM, Hertz MI. The registry of the International Society for Heat and Lung Transplantation: twenty-first official adult heart transplant report-2004. J Heart Lung Transplant. 2004;23: 796-803.

2. Radovancevic B, McGiffin DC, Kobashigawa JA, et al. Retransplantation in 7,290 primary transplant patients: a 10-year multiinstitutional study. J Heart Lung Transplant. 2003;22:862-8.

3. Sipahi I, Starling RC. Cardiac allograft vasculopathy: an update. Heart Fail Clin. 2007;3:87-95.

4. Mehra MR. Contemporary concepts in prevention and treatment of cardiac allograft vasculopathy. Am J Transplant. 2006;6:1248-56.

5. Gao SZ, Alderman EL, Schroeder JS, Silverman JF, Hunt SA. Accelerated coronary vascular disease in the heart transplant patient: coronary arteriographic findings. J Am Coll Cardiol. 1988;12:334-40.

6. Mancini D, Pinney S, Burkhoff D, et al. Use of rapamycin slows progression of cardiac transplantation vasculopathy. Circulation. 2003;108:48-53.

7. Keogh A, Richardson M, Ruygrok P, et al. Sirolimus in de novo heart transplant recipients reduces acute rejection and prevents coronary artery disease at 2 years: a randomized clinical trial. Circulation. 2004;110:2694-700.

8. Eisen HJ, Tuzcu EM, Dorent R, et al. Everolimus for the prevention of allograft rejection and vasculopathy in cardiac-transplant recipients. N Engl J Med. 2003; 349:847-58.

9. Frazier OH, Tuzun E, Eichstadt H, et al. Transmyocardial laser revascularization as an adjunct to coronary artery bypass grafting: a randomized, multicenter study with 4-year follow-up. Tex Heart Inst J. 2004;31:231-9.

10. Mehra MR, Uber PA, Prasad AK, et al. Long-term outcome of cardiac allograft vasculopathy treated by transmyocardial laser revascularization: early rewards, late losses. J Heart Lung Transplant. 2000;19:801-4.

11. Halle AA 3rd, DiSciascio G, Massin EK, et al. Coronary angioplasty, atherectomy and bypass surgery in cardiac transplant recipients. J Am Coll Cardiol. 1995;26: 120-8.

12. Parry A, Roberts M, Parameshwar J, Wallwork J, Schofield P, Large S. The management of post-cardiac transplantation coronary artery disease. Eur J Cardiothorac Surg. 1996;10:528-32; discussion 553.

13. Musci M, Loebe M, Wellnhofer E, et al. Coronary angioplasty, bypass surgery, and retransplantation in cardiac transplant patients with graft coronary disease. Thorac Cardiovasc Surg. 1998;46:268-74.

14. Nader ND, Khadra WZ, Reich NT, Bacon DR, Salerno TA, Panos AL. Blood product use in cardiac revascularization: comparison of on- and off- pump techniques. Ann Thorac Surg. 1999;1640-3. 